\title{
Poly(Alkylene 2,5-Thiophenedicarboxylate) Polyesters: A New Class of Bio-Based High-Performance Polymers for Sustainable Packaging
}

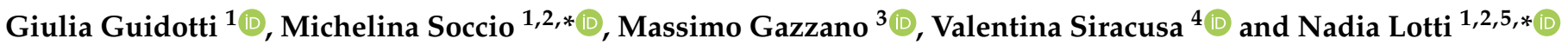 \\ 1 Department of Civil, Chemical, Environmental and Materials Engineering, University of Bologna, \\ Via Terracini 28, 40131 Bologna, Italy; giulia.guidotti9@unibo.it \\ 2 Interdepartmental Center for Industrial Research on Advanced Applications in Mechanical Engineering and \\ Materials Technology, CIRI-MAM, University of Bologna, 40126 Bologna, Italy \\ 3 Institute of Organic Synthesis and Photoreactivity, ISOF-CNR, Via Gobetti 101, 40129 Bologna, Italy; \\ massimo.gazzano@isof.cnr.it \\ 4 Department of Chemical Science, University of Catania, Viale A. Doria 6, 95125 Catania, Italy; \\ vsiracus@dmfci.unict.it \\ 5 Interdepartmental Center for Agro-Food Research, CIRI-AGRO, University of Bologna, 40126 Bologna, Italy \\ * Correspondence: m.soccio@unibo.it (M.S.); nadia.lotti@unibo.it (N.L.)
}

check for updates

Citation: Guidotti, G.; Soccio, M.; Gazzano, M.; Siracusa, V.; Lotti, N. Poly(Alkylene

2,5-Thiophenedicarboxylate)

Polyesters: A New Class of Bio-Based High-Performance Polymers for Sustainable Packaging. Polymers 2021, 13, 2460. https://doi.org/10.3390/ polym 13152460

Academic Editor: Tzong-Ming Wu

Received: 15 June 2021

Accepted: 20 July 2021

Published: 27 July 2021

Publisher's Note: MDPI stays neutral with regard to jurisdictional claims in published maps and institutional affiliations.

Copyright: (c) 2021 by the authors. Licensee MDPI, Basel, Switzerland. This article is an open access article distributed under the terms and conditions of the Creative Commons Attribution (CC BY) license (https:// creativecommons.org/licenses/by/ $4.0 /)$.

\begin{abstract}
In the present study, 100\% bio-based polyesters of 2,5-thiophenedicarboxylic acid were synthesized via two-stage melt polycondensation using glycols containing 3 to 6 methylene groups. The so-prepared samples were characterised from the molecular point of view and processed into free-standing thin films. Afterward, both the purified powders and the films were subjected to structural and thermal characterisation. In the case of thin films, mechanical response and barrier properties to $\mathrm{O}_{2}$ and $\mathrm{CO}_{2}$ were also evaluated. From the results obtained, it emerged that the length of glycolic sub-units is an effective tool to modulate the chain mobility and, in turn, the kind and amount of ordered phases developed in the samples. In addition to the usual amorphous and $3 \mathrm{D}$ crystalline phases, in all the samples investigated it was possible to evidence a further phase characterised by a lower degree of order (mesophase) than the crystalline one, whose amount is strictly related to the glycol sub-unit length. The relative fraction of all these phases is responsible for the different mechanical and barrier performances. Last, but not least, a comparison between thiophene-based homopolymers and their furan-based homologues was carried out.
\end{abstract}

Keywords: 2,5-thiophenedicarboxylic acid; thermal properties; barrier properties; mechanical properties; 2D-ordered structure; structure-property relationship

\section{Introduction}

Plastic waste has become a matter of great concern in recent years and is pushing governments and society to come up with new strategies to face the problem. If the amount of both plastic waste volume and the exploitation of non-renewable sources are analysed, it is immediately clear how the development of sustainable alternatives to traditional plastics is urgent and re-use, where possible, should be encouraged. If it is true that since 2006, the amount of plastic waste recycled has doubled, it is also likely that we are still far from the so called "zero landfilling" needed to achieve the circular economy of plastics, as in many cases plastic waste is sent to landfills or, even worse, is disposed of in marine and terrestrial environments [1].

It is also important to consider that among the principal current applications of plastics, packaging alone covers $39.9 \%$ of them [1]: as far as packaging is concerned, petrochemicalbased ones are currently largely used thanks to its abundance, great mechanical strength, and barrier properties together with low production costs despite most of it being nonbiodegradable. As an alternative, plastics obtained from renewable sources can represent a 
valuable solution thanks to their resource efficiency and reduced carbon footprint. This potential is confirmed also by the increasing bioplastics market size and demand: according to the most recent studies, global bioplastics production capacity is estimated to grow from 2.11 million tons in 2019 to about 2.43 million tons in 2024 [2]. However, to date there are still some important drawbacks related to the final properties of these bioplastics, limiting their current applications, such as short-term stability, bad processability, poor mechanical strength, brittleness, or relatively high gas permeability.

Besides the use of renewable sources, also reducing packaging volumes could be a very practical route to reduce environmental impact. For this reason, many efforts have been devoted to the research of optimized flexible solutions in place of rigid and oversized ones, keeping at the same time the pack quality and the product protection, in particular when the product is characterised by a very short life cycle, as in the case of food. For example, vacuum skin packs can provide excellent barrier properties, minimizing volumes while at the same time preserving the product's shelf life. Another issue is related to multilayer materials, commonly used to better preserve food, in which high barrier properties are ensured by the combination of different layers. These strata are intimately overlapped and very thin, and thus very difficult to separate in view of a possible reuse. Therefore, the use of monomaterials, which can provide an effective barrier to gases on one side and can be also easily recovered after use, should be preferred.

Aware of the severity and the complexity of this scenario, the European Commission has adopted both a Waste Framework Directive (2008/98/EC) and an action plan for a circular economy in view of ensuring that all plastic packaging would be recyclable by 2030 [3]. In addition, the U.S. Department of Energy has identified twelve building blocks that can be produced from renewable sources, such as sugars, which can in turn be converted to many high-value bio-based chemicals [4]. Among them, 2,5-furandicarboxylic acid (FDCA) is of particular interest, both academically and industrially, since one of its derived materials, poly(ethylene furanoate) (PEF), is considered the most credible green alternative of the fossil-based poly(ethylene terephthalate) (PET), which is largely employed in packaging applications. Moreover, according to published studies, PEF is more performant than PET in terms of mechanical and barrier response [5-9]. It is known that FDCA can be obtained from sugar dehydration, obtaining 5 hydroxymethyl-furfural (HMF) as an intermediate product [10], with high yield and purity.

In recent years, 2,5-thiophenedicarboxylic acid (2,5-TDCA) has attracted growing attention due to its chemical structure, which is similar to that of FDCA. In addition, 2,5-TDCA can be derived from renewable sources and is already industrially produced from adipic acid, which can be obtained, in turn, from glucaric acid, muconic acid, or lignin [11-14]. As proof of the growing interest in these polyesters, many scientific studies have been recently published, including those conducted outside of our research group, demonstrating the great potential of both thiophene-based homopolymers and copolymeric systems [15-24].

Moreover, since the enzymatic degradability of PET and PEF has already been demonstrated [25-27], more recently the biodegradability of poly(butylene 2,5-furandicarboxylate) PBF and poly(butylene 2,5-thiophenedicarboxylate) PBTF was also investigated. It was proved that Humicola insolens (HiC) and Thermobifida cellulosilytica (Cut) cutinases can hydrolyse both homopolymers, opening a new way for the industrial recycling of furanand thiophene-based materials [28].

Considering the above-described scenario, the present work aimed to synthesize by two-step polycondensation four fully bio-based poly (alkylene 2,5-thiophenedicarboxylate)s, starting from dimethyl 2,5-thiophenedicarboxylate, the dimethyl ester of 2,5-TDCA, and glycols of different length (the number of methylene groups changed from 3 to 6). These can be all derived from renewable sources as well as 2,5-TDCA. By acting only on the number of $\mathrm{CH}_{2}$ groups in the glycol moiety, it was possible to cover a wide range of properties, making the materials obtained particularly suitable for the realisation of rigid as well as flexible films for packaging applications. The polymers synthesized, in the 
form of both powders and thin films, were characterised from the molecular, structural, thermal, mechanical, and gas barrier point of view, and correlations between structure and properties were extrapolated. In addition, the presence of a mesophase was hypothesized, and both the amount of ordered domains developed and the mechanism of their formation was compared to those of furan-based analogues [29].

\section{Materials and Methods}

\subsection{Materials}

2,5-thiophenedicarboxylic acid (2,5-TDCA) was purchased from TCI (Tokyo, Japan), titanium tetrabutoxide (TBT), titanium isopropoxide (TIP), 1,3-propanediol (PD), 1,4-butanediol (BD), 1,5-pentanediol (PeD), and 1,6-hexanediol (HD) were purchased from Sigma-Aldrich (Milan, Italy). All reagents were used as received.

\subsection{Synthesis}

Prior to polymer synthesis, dimethyl 2,5-thiophenedicarboxylate (DMTF) was prepared, starting from 2,5-TDCA, according to the procedure described in [18]. Afterward, all the homopolymers were synthetized through two-stage melt polycondensation, starting from DMTF (0.02 mol, $4 \mathrm{~g}$ ) and $\mathrm{PD} / \mathrm{BD} / \mathrm{PeD} / \mathrm{HD}(0.04 \mathrm{~mol}$, corresponding to $3.04 \mathrm{~g}$ for $\mathrm{PD}, 3.60 \mathrm{~g}$ for $\mathrm{BD}, 4.17 \mathrm{~g}$ for PeD and $4.73 \mathrm{~g}$ for HD, respectively), using TBT and TTIP as catalysts (200 ppm of each). In all cases, a diester:glycol molar ratio of 1:2 was used in order to favour the DMTF solubilisation. The syntheses were carried out in a glass reactor put in a termostated bath. The reaction mixture was kept under continuous stirring by using a two-bladed centrifugal stirrer connected to an overhead motor. Syntheses proceeded in two stages. The first one occurred under pure nitrogen flow at a temperature of $180^{\circ} \mathrm{C}$. This condition was maintained until $90 \%$ of the theoretical amount of methanol was distilled off (about $2 \mathrm{~h}$ from the solubilisation of the dimethyl ester). During the second stage, pressure was reduced gradually to $0.06 \mathrm{mbar}$ and temperature raised up to $220^{\circ} \mathrm{C}$ to favour the removal of the glycolic excess as well as the increasing of molecular weight. Polymerisation was stopped when a constant torque value was reached (after about two additional hours).

Prior to solid-state polymer characterisation, all the samples were purified to remove impurities, oligomers, and catalysts. The polyesters were first dissolved in chloroform (in the case of PPTF some drops of hexafluoro-2-propanol were needed for the complete polymer solubilisation), then precipitated in methanol. Lastly, the purified powders were kept under a vacuum at room temperature for $48 \mathrm{~h}$ to remove the residual solvent.

\subsection{Molecular Characterisation}

Chemical structure was confirmed by means of proton nuclear magnetic resonance $\left({ }^{1} \mathrm{H}\right.$ NMR), employing a Varian Inova $400 \mathrm{MHz}$ instrument (Palo Alto, CA, USA). More in detail, each homopolymer was dissolved in deuterated chloroform containing 0.03 vol. $\%$ tetramethylsilane (TMS) as an internal standard (solutions' concentration was about $0.5 \mathrm{wt} \%$ ). For PPTF, a mixture of trifluoracetic acid and chloroform was used $(20 \% v / v)$. The spectra were recorded at room temperature with a relaxation delay of $1 \mathrm{~s}$, acquisition time of $1 \mathrm{~s}$ and up to 64 repetitions.

In order to determine the molecular weight of the synthesized materials, gel permeation chromatography (GPC) was performed at $30^{\circ} \mathrm{C}$ using an $1100 \mathrm{HPLC}$ system (Agilent Technologies, Santa Clara, CA, USA) equipped with a PLgel 5-mm MiniMIX-C column. A UV detector was employed, and chloroform was used as an eluent. We used $0.3 \mathrm{~mL} / \mathrm{min}$ flow and sample concentrations of about $2 \mathrm{mg} / \mathrm{mL}$. Polystyrene standards in the range of $800-100,000 \mathrm{~g} / \mathrm{mol}$ were used to obtain a calibration curve.

\subsection{Film by Compression Moulding}

Polymeric films of about $100 \mu \mathrm{m}$ thickness were prepared via compression moulding using a Carver laboratory press (Wabash, IN, USA). The PPTF, PBTF, PPeTF and PHTF samples were melted at temperatures of $205,180,95$ and $125^{\circ} \mathrm{C}$, respectively, between 
two Teflon sheets. Therefore, a pressure of $7 \mathrm{ton} \cdot \mathrm{m}^{-2}$ was applied for $2 \mathrm{~min}$. The samples were then ballistically cooled down to room temperature in press, the process taking about $15 \mathrm{~min}$. The as-obtained film pictures are shown in Figure S1. Prior to further tests, the films of the polymers with a glass transition temperature below room temperature (PPeTF and PHTF) were stored at room temperature for 3 weeks in order to let them reach equilibrium crystallinity.

\subsection{Thermal and Structural Characterisation}

Thermal stability and thermal transitions of the homopolymers were investigated by means of thermogravimetric analysis (TGA) and calorimetric measurements (DSC), respectively. In the former case, the analyses were carried out using a Perkin Elmer TGA7 apparatus (gas flow: $40 \mathrm{~mL} / \mathrm{min}$ ), under an inert $\mathrm{N}_{2}$ atmosphere (Shelton, $\mathrm{CT}, \mathrm{USA}$ ). Weighed samples of about $10 \mathrm{mg}$ were heated at a constant rate $\left(10^{\circ} \mathrm{C} / \mathrm{min}\right)$ from $40{ }^{\circ} \mathrm{C}$ up to $800^{\circ} \mathrm{C}$. In this way, it was possible to calculate the temperature corresponding to initial degradation $\left(\mathrm{T}_{\text {onset }}\right)$ as well as the temperature at which maximum weight loss occurs $\left(\mathrm{T}_{\max }\right)$.

As for calorimetric measurements, these ones were carried out on both purified powders and films using a Perkin Elmer DSC6 (Shelton, CT, USA) calibrated with indium and cyclohexane standards. As is known, DSC analysis allows the determination of glass transition temperature $\left(T_{g}\right)$, melting and crystallisation temperatures $\left(T_{m}\right.$ and $T_{c c}$, respectively), as well as the specific heat increment associated with the glass transition of the amorphous phase $\left(\Delta \mathrm{c}_{\mathrm{p}}\right)$, and the heat of fusion and crystallisation $\left(\Delta \mathrm{H}_{\mathrm{m}}\right.$ and $\Delta \mathrm{H}_{\mathrm{cc}}$, respectively), relative to the crystalline portion of the polymer. $T_{g}$ was calculated as the midpoint of the heat capacity increment related to the glass-to-rubber transition. $T_{m}$ and $\mathrm{T}_{\mathrm{cc}}$ were considered as the peak values of the endothermal and exothermal phenomena in the DSC curve, respectively, $\Delta c_{p}$ was calculated from the vertical distance between the two extrapolated baselines at $\mathrm{T}_{\mathrm{g}}$, while $\Delta \mathrm{H}_{\mathrm{m}}$ and $\Delta \mathrm{H}_{\mathrm{cc}}$ were calculated considering the global area subtended by melting and crystallisation peaks, respectively. In the procedure adopted, polymeric samples of about $8 \mathrm{mg}$ were put in aluminium pans and heated at a constant rate $\left(20^{\circ} \mathrm{C} / \mathrm{min}\right)$ from $-50^{\circ} \mathrm{C}$ to $210,200,160$ and $180{ }^{\circ} \mathrm{C}$ for PPTF, PBTF, PPeTF and PHTF, respectively, held there for $3 \mathrm{~min}$, and then rapidly cooled $\left(100{ }^{\circ} \mathrm{C} / \mathrm{min}\right)$ to $-50{ }^{\circ} \mathrm{C}$. They were then heated again at the same heating rate (II scan).

Wide-angle X-ray scattering (WAXS) patterns of purified powders and films were recorded by means of a PANalytical X'PertPro diffractometer (Almelo, The Netherlands) equipped with a fast solid-state $X^{\prime}$ Celerator detector and a copper target $(\lambda=0.1548 \mathrm{~nm})$. The samples were scanned in the range $2 \theta=5^{\circ}$ to $60^{\circ}$ (step size of $0.10^{\circ}$, acquisition time of $100 \mathrm{~s}$ per step). The degree of crystallinity $\left(\mathrm{X}_{\mathrm{c}}\right)$ was calculated dividing the value of the crystalline diffraction area $\left(A_{c}\right)$ by the total area of the diffraction pattern $\left(A_{t}\right), X_{c}=A_{c} / A_{t}$. $A_{c}$ was determined by subtracting the amorphous halo from the $A_{t}$ value itself.

\subsection{Mechanical Characterisation}

Tensile tests were carried out on rectangular films $(5 \mathrm{~mm} \times 50 \mathrm{~mm}$, gauge length of $20 \mathrm{~mm}$ ) by means of an Instron 5966 testing machine (Norwood, MA, USA) equipped with a rubber grip and a $1 \mathrm{KN}$ load cell. Experiments were performed at room temperature with a crosshead speed of $10 \mathrm{~mm} / \mathrm{min}$, testing at least seven specimens for each polymeric sample. The so-obtained load-displacement curves were then converted to stress-strain curves. The value of the tensile elastic modulus (E) was calculated from the initial linear slope of the stress-strain curve. The values of stress at break $\left(\sigma_{\mathrm{B}}\right)$ and elongation at break $\left(\varepsilon_{\mathrm{B}}\right)$ were also determined. The results are reported as the average value \pm standard deviation.

\subsection{Gas Barrier Properties Evaluation}

In order to evaluate gas transmission rates through polymeric films, permeability tests were performed by using a manometric method (Permeance Testing Device, type GDP-C (Brugger Feinmechanik GmbH, München, Germany)), in accordance with ASTM 
1434-82, DIN 53 536, ISO/DIS 15 105-1 and the gas permeability testing manual of the instrument. Each circular film (film area of $78.5 \mathrm{~cm}^{2}$ ) was placed in between two chambers. First, the system was put under high vacuum, and then the upper chamber was filled with the selected gas $\left(\mathrm{CO}_{2}\right.$ or $\mathrm{O}_{2}$ food grade, $\left.0 \% \mathrm{RH}\right)$ at $23{ }^{\circ} \mathrm{C}$ and atmospheric pressure, with a gas stream of $100 \mathrm{~cm}^{3} / \mathrm{min}$. Any variation in gas pressure was recorded, as a function of time, by a pressure sensor located in the lower chamber. Gas transmission rate (GTR $\left[\mathrm{cm}^{3} \mathrm{~cm} \mathrm{~m}^{-2} \mathrm{~d}^{-1} \mathrm{~atm}^{-1}\right]$ ), i.e., the value of film permeability, was determined taking into account the pressure increase as a function of time and of the whole volume of the device. The GTR values were normalised for the film thickness, the latter measured as the average value among five different measurements.

\section{Results and Discussion}

\subsection{Synthesis and Molecular Characterisation}

The chemical structure of the homopolyesters under investigation are reported in Figure 1 , together with a schematic representation of the synthetic process. All the materials were characterised by a diacid aromatic subunit derived from 2,5-TDCA and by an aliphatic glycolic subunit differing for the number of methylene groups ranging from 3 to 6 . It is worth noting from the chemical structure a nonpolar moiety, coming from the aliphatic segment, was combined with a polar one, i.e., thiophene ring, whose dipole moment $(\mu)$ was $0.51 \mathrm{D}$. The starting 2,5-TDCA was esterified because the impurities present in the diacid acting as nucleating agents affected the polymer microstructure and consequently the final polymer properties, such as the mechanical and gas barrier ones [18]. The assynthesized polymers looked slightly coloured due to the presence of residual catalysts, while after purification, in all cases, white powders were obtained.
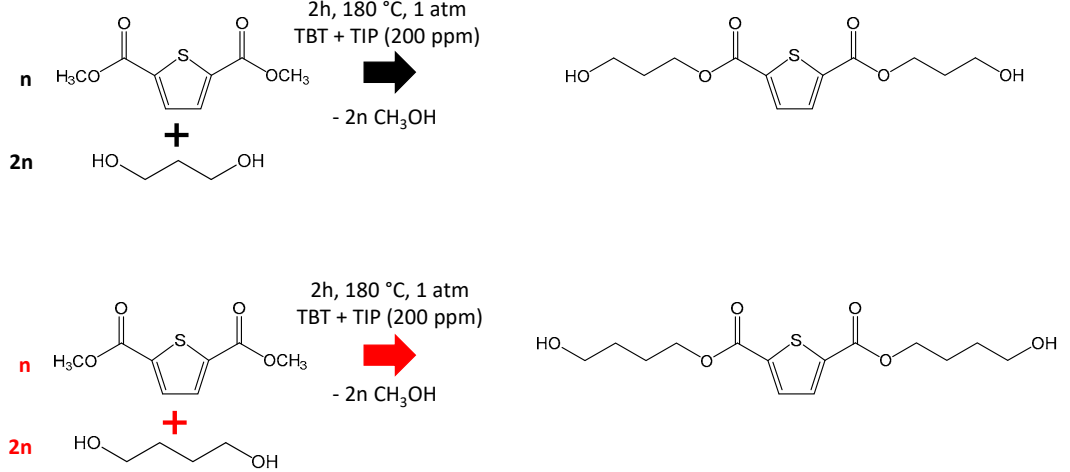
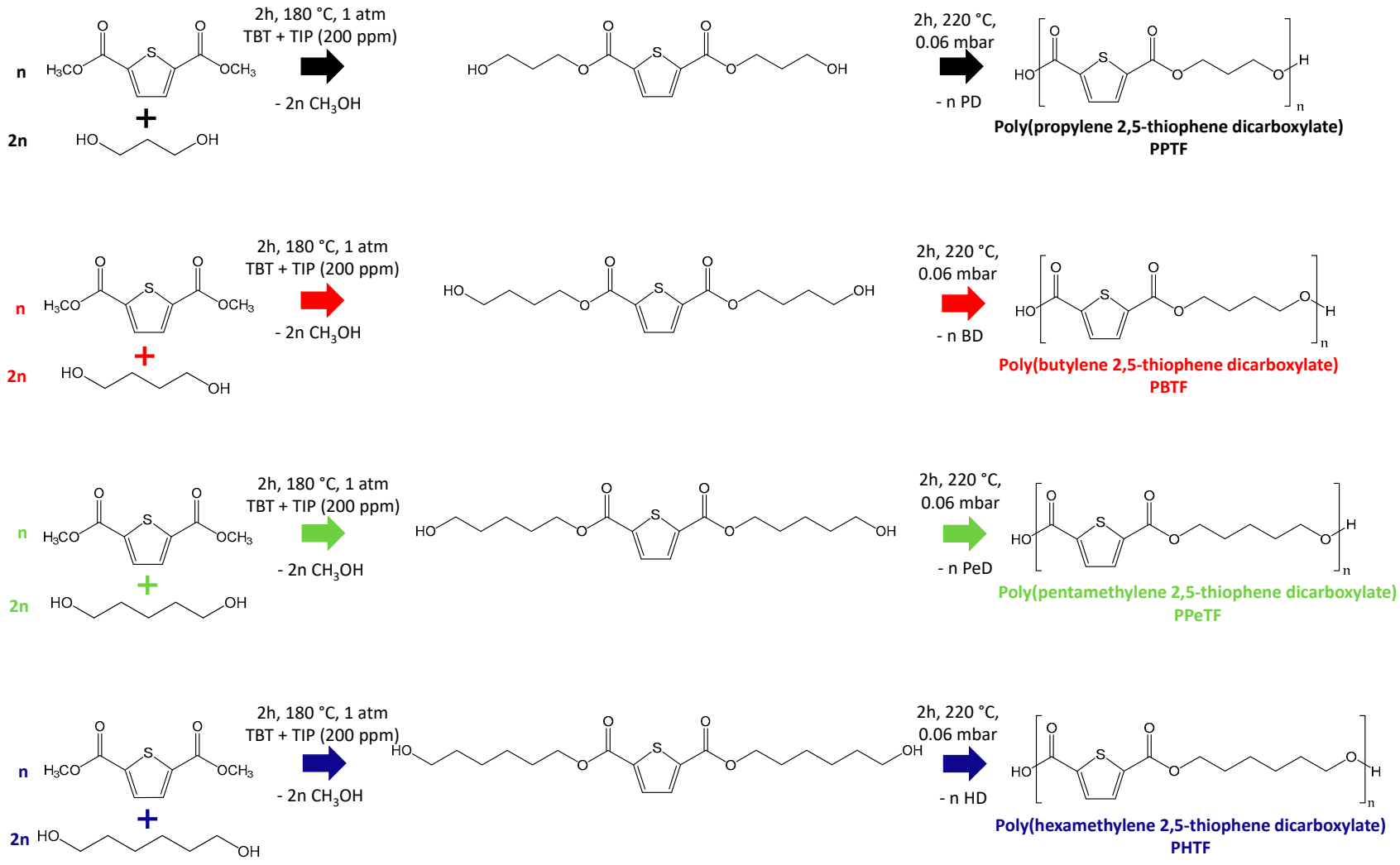

Figure 1. Schematic representation of the synthetic process together with the chemical structure of the homopolyesters under study: PPTF, PBTF, PPeTF and PHTF. 
The chemical structures were confirmed via ${ }^{1} \mathrm{H}-\mathrm{NMR}$ spectroscopy (Figure 2). Table 1 collects the peak position and multiplicity observed in the NMR spectra. No extra signals were found, proving the absence of impurities in the synthesized samples.
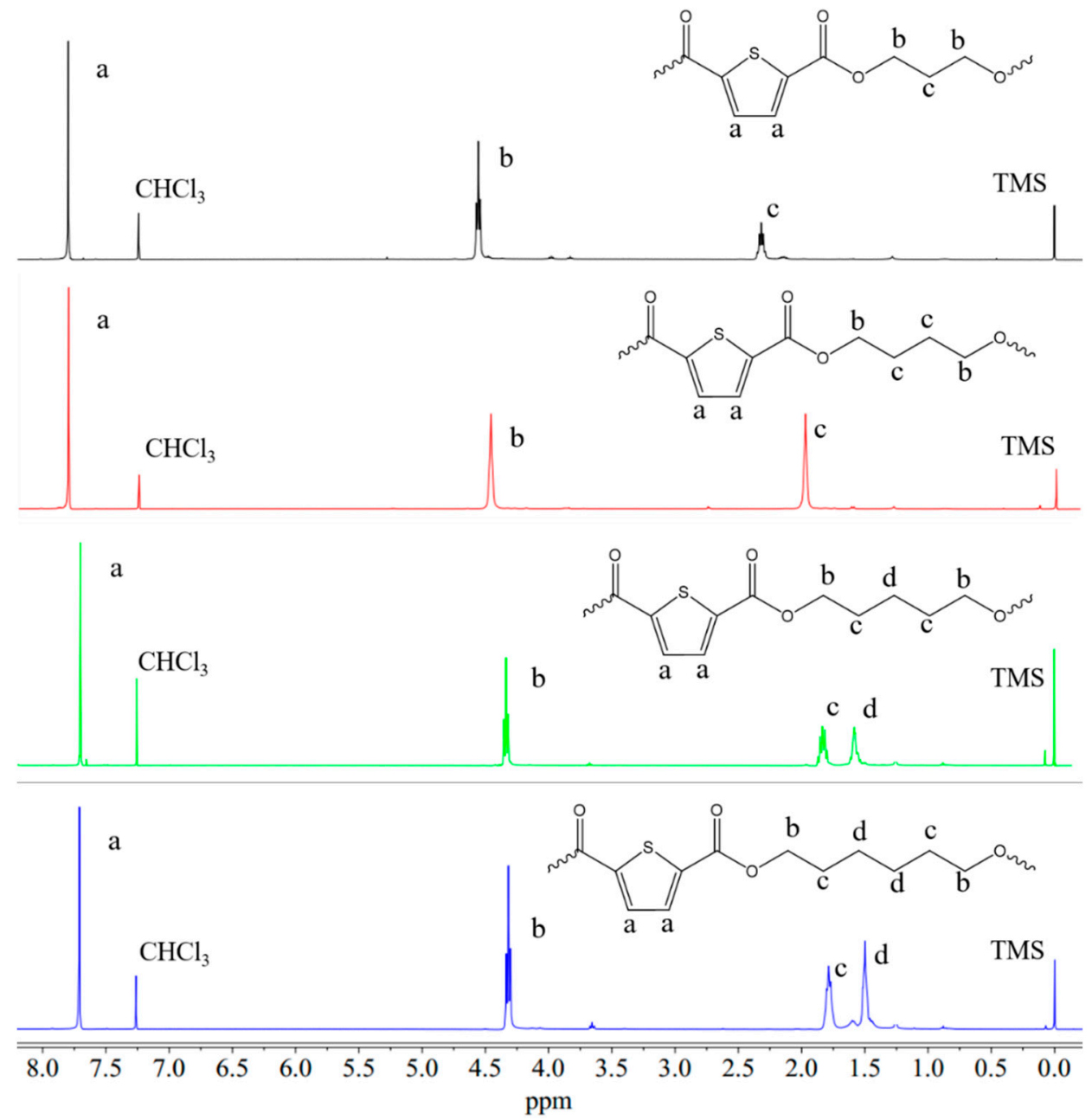

Figure 2. ${ }^{1} \mathrm{H}-\mathrm{NMR}$ spectra of the homopolymers under study together with peaks' assignment.

Table 1. ${ }^{1} \mathrm{H}-\mathrm{NMR}$ peak position and molecular characterisation data for the homopolymers under study.

\begin{tabular}{|c|c|c|c|c|}
\hline & \multicolumn{2}{|c|}{ Peak Position (ppm) } & \multirow{2}{*}{$\begin{array}{l}M_{n} \\
(\mathrm{Da})\end{array}$} & \multirow{2}{*}{$\mathbf{D}$} \\
\hline & Diacid Subunit & Glycolic Subunit & & \\
\hline PPTF & $7.83(2 \mathrm{H}, \mathrm{s})$ & $\begin{array}{c}2.34(2 \mathrm{H}, \mathrm{m}) \\
4.60(4 \mathrm{H}, \mathrm{t})\end{array}$ & 26,300 & 2.3 \\
\hline PBTF & $7.83(2 \mathrm{H}, \mathrm{s})$ & $\begin{array}{c}1.99(4 \mathrm{H}, \mathrm{m}) \\
4.48(4 \mathrm{H}, \mathrm{t}) \\
1.58(2 \mathrm{H}, \mathrm{m})\end{array}$ & 39,600 & 2.0 \\
\hline PPeTF & $7.70(2 \mathrm{H}, \mathrm{s})$ & $\begin{array}{c}1.82(4 \mathrm{H}, \mathrm{m}) \\
4.34(4 \mathrm{H}, \mathrm{t}) \\
1.51(4 \mathrm{H}, \mathrm{m})\end{array}$ & 25,800 & 2.5 \\
\hline PHTF & $7.70(2 \mathrm{H}, \mathrm{s})$ & $\begin{array}{c}1.81(4 \mathrm{H}, \mathrm{m}) \\
4.32(4 \mathrm{H}, \mathrm{t})\end{array}$ & 32,400 & 2.3 \\
\hline
\end{tabular}

All the materials analysed show high and comparable values of $\mathrm{M}_{\mathrm{n}}$ (see Table 1 and the chromatograms shown in Figure S2) and a polydispersity index (D) around 2, typical 
of polyesters obtained by melt polycondensation, corroborating the optimization of the polymerisation process.

\subsection{Thermal Characterisation}

\subsubsection{Powder Samples}

The first scan DSC curves of powder samples are shown in Figure 3A and the relative data are displayed in Table S1. The DSC traces of all samples present the typical profile of semicrystalline materials with an endothermic baseline deviation related to glass-to-rubber transition, followed by an endothermic phenomenon associated with the melting of crystalline phase. This phase behaviour can be explained due to solvent-induced crystallisation. In PPTF DSC trace, the glass transition phenomenon is not detectable because of its very low intensity due to the high crystallinity degree. Besides the main melting peak, two low and broad endothermic signals can be identified around $60-70^{\circ} \mathrm{C}$, whose origin is not so clear. Broad multiple melting peaks were also detected for PBTF, PPeTF and PHTF samples, suggesting melting-crystallisation-re-melting processes occurring during heating scan or in the presence of different crystalline phases.
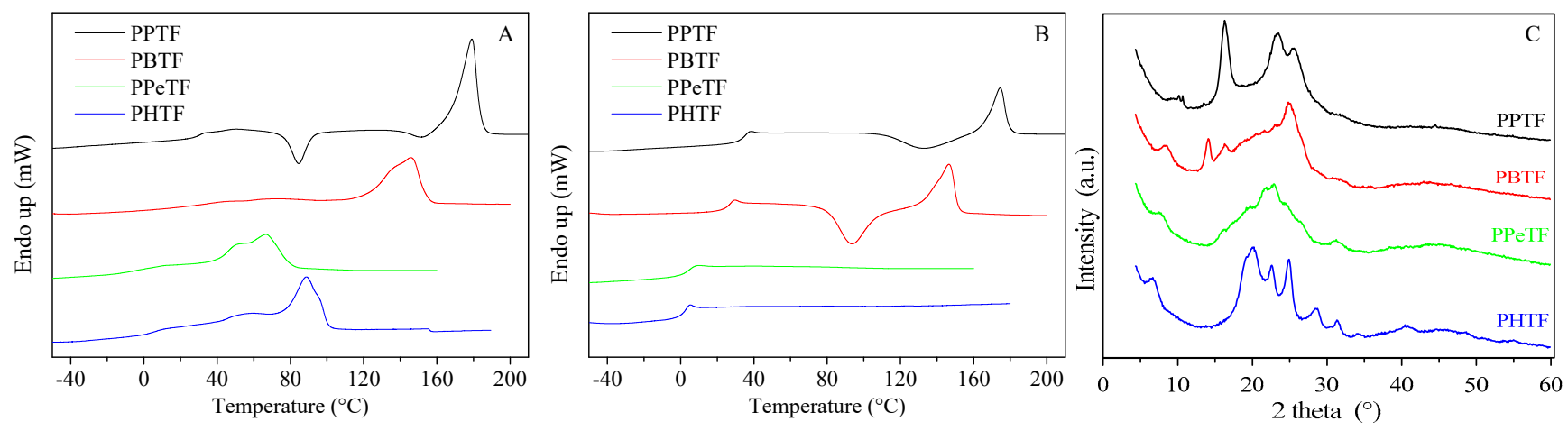

Figure 3. (A) I scan; (B) II scan DSC curves; (C) WAXS profiles of PPTF, PBTF, PPeTF and PHTF purified powders.

In general, the position of the main endotherm $\left(T_{m}\right)$ moved toward lower temperature as the glycol length increased, with the exception of PPeTF, which exhibited the lowest melting temperature.

As is well known, semicrystalline polymers behave differently from their completely amorphous analogues due to the physical crosslinks provided by crystalline structures, which limit chain mobility, raising the $T_{g}$ value $[30,31]$. Therefore, we decided to perform a DSC scan after rapid cooling from the molten state with the aim of quenching the polymer macromolecules. The relative curves are reported in Figure 3B, while the corresponding calorimetric data are displayed in Table S1. The results demonstrated the effectiveness of the rapid cooling from the melt in quenching the powders, being all the materials amorphous in the II scan. Nevertheless, some differences in the crystallisation capability from the glass could be evidenced. In particular, in the second run PPeTF and PHTF showed just the endothermic jump associated with the glass-to-rubber transition, while PPTF and PBTF powders in addition to the $T_{g}$ step presented an endothermic signal together with an exothermic one of equal intensity, demonstrating their chains are capable of rearranging into an ordered structure upon heating. However, since $\Delta \mathrm{H}_{\mathrm{c}} \approx \Delta \mathrm{H}_{\mathrm{m}}$, one can assert rapid cooling from the melt was effective in quenching both PPTF and PBTF powders. Considering the $T_{g}$ values, as expected, the higher the number of methylene groups the higher the flexibility of macromolecular chains, i.e., the lower the $T_{g}$ value. Moreover, a longer glycol subunit implies fewer stiff aromatic rings along the polymer backbone. More in detail, PPTF was the only glassy material at room temperature, being its $\mathrm{T}_{\mathrm{g}}>\mathrm{RT}$. Conversely, PPeTF and PHTF were in the rubbery state, with $\mathrm{T}_{\mathrm{g}}$ well below room temperature. PBTF was instead characterised by a glass transition temperature near room temperature. 
WAXS patterns of powder samples are reported in Figure 3C. At first glance, it is clear that the samples were characterised by different crystallinity degrees, $X_{c}$ being equal to $29 \%, 16 \%, 9 \%$ and $25 \%$ for PPTF, PBTF, PPeTF and PHTF, respectively. The profiles showed different shapes with a variation of peak positions on the 2-theta scale and intensities peculiar for each sample. This suggests the crystal phases probably do not share any similarities, i.e., are not isomorphous. By comparing with previous reported data [15], we can ascribe the crystal fraction of the PPTF sample to its $\beta$-form. Indeed, the four main peaks at $9.3^{\circ}, 16.3^{\circ}, 23.3^{\circ}$ and $25.5^{\circ}$ were previously reported for a film sample named $\beta$-PPTF [15]. As for the PBTF sample, the observed main peaks at $8.2^{\circ}, 14.0^{\circ}, 16.2^{\circ}$ and $24.8^{\circ}$ permitted us to recognize in the PBTF powder the $\gamma$-phase [18]. PPeTF showed a very low crystallinity degree with peaks, or better peak clues, at $7.6^{\circ}, 16.2^{\circ}, 21.6^{\circ}, 22.8^{\circ}$, $24.3^{\circ}$ and $31.1^{\circ}$. The PHTF profile was characterised by well-defined peaks identified at $6.5^{\circ}, 19.2^{\circ}, 20.0^{\circ}, 22.6^{\circ}, 24.9^{\circ}, 28.6^{\circ}$ and $31.3^{\circ}$, indicating the presence of a conspicuous amount of crystals. This pattern is compatible with the one reported by authors of another study [19].

\subsubsection{Compression-Moulded Films}

Prior to solid-state characterisation, compression-moulded films were subjected to 3 weeks storage at room temperature to uniform their thermal history since both PPeTF and PHTF are characterised by $\mathrm{T}_{\mathrm{g}} \mathrm{s}$ below room temperature, and the $\mathrm{T}_{\mathrm{g}}$ of PBTF is near RT.

Firstly, compression-moulded films (Figure S1) were subjected to thermogravimetric analysis (TGA) under pure nitrogen flux. The temperatures of initial degradation ( $\mathrm{T}_{\text {onset }}$ ) and of maximum weight loss rate $\left(\mathrm{T}_{\max }\right)$ are displayed in Table 2, while the corresponding TGA curves and derivatives are shown in Figure 4A,B, respectively. All the polyesters displayed very good thermal stability, with $\mathrm{T}_{\text {onset }}$ above $370{ }^{\circ} \mathrm{C}$ and $\mathrm{T}_{\max }$ above $400{ }^{\circ} \mathrm{C}$, as clearly evidenced by thermograms' derivatives, the degradation process occurring in all cases in two steps, the main one around $400{ }^{\circ} \mathrm{C}$. The second step was sensibly smaller in all cases and occurred immediately afterward. Lastly, no residual mass at $800{ }^{\circ} \mathrm{C}$ was found. Some differences among the samples could be found. PPTF turned out to be the fastest-degrading material, presumably because of the high amount of ester groups, which undergo thermal cleavage more easily, and the presence of 1,3-propanediol, which favours $\beta$-scission reactions as previously observed for other polyesters, among these PPF, its furan-based counterpart [29,32]. PBTF was, on the contrary, the most thermally stable among the family, while PPeTF and PHTF were characterised by a very similar thermal stability, intermediate between those of PPTF and PBTF. This trend was slightly different from the one observed in the case of furan-based materials, where PPeF was the most thermally stable [33].

Each thiophene-based polyester was directly compared with its furan-based counterpart (see Figure 4C). As you can see, PPTF and PBTF appeared to be more thermally stable than their furan-based counterparts (PPF and PBF). We hypothesize such a result could be due to the higher resonance energy, $\mathrm{p}$-to-d $\pi$-back bonding, and lack of ring strain due to the longer $\mathrm{C}-\mathrm{S}$ bond of the thiophene ring. Conversely, poly(pentamethylene furanoate) (PPeF) turned out to be more thermally stable than PPeTF, whereas PHF and PHTF showed very similar thermal stability.

Table 2. Thermal characterisation (TGA and DSC) data of the homopolymers under study in the form of compressionmoulded films.

\begin{tabular}{|c|c|c|c|c|c|c|c|c|c|c|c|c|c|c|}
\hline & \multirow[b]{2}{*}{$\underset{{ }^{\circ} \mathrm{C}}{\mathrm{T}_{\text {onset }}}$} & \multirow[b]{2}{*}{$\begin{array}{c}\mathrm{T}_{\max } \\
{ }^{\circ} \mathrm{C}\end{array}$} & \multicolumn{6}{|c|}{ I Scan } & \multicolumn{6}{|c|}{ II Scan } \\
\hline & & & $\begin{array}{l}\mathrm{T}_{\mathrm{g}} \\
{ }^{\circ} \mathrm{C}\end{array}$ & $\begin{array}{c}\Delta c_{p} \\
J / g^{\circ} \mathrm{C}\end{array}$ & $\begin{array}{l}\mathrm{T}_{\mathrm{cc}} \\
{ }^{\circ} \mathrm{C}\end{array}$ & $\begin{array}{c}\Delta \mathbf{H}_{\mathrm{cc}} \\
\mathrm{J} / \mathrm{g}\end{array}$ & $\begin{array}{l}\mathrm{T}_{\mathrm{m}} \\
{ }^{\circ} \mathrm{C}\end{array}$ & $\begin{array}{c}\Delta \mathbf{H}_{\mathrm{m}} \\
\mathrm{J} / \mathrm{g}\end{array}$ & $\begin{array}{l}\mathrm{T}_{\mathrm{g}} \\
{ }^{\circ} \mathrm{C}\end{array}$ & $\begin{array}{c}\Delta \mathrm{c}_{\mathrm{p}} \\
\mathrm{J} / \mathrm{g}^{\circ} \mathrm{C}\end{array}$ & $\begin{array}{l}\mathrm{T}_{\mathrm{cc}} \\
{ }^{\circ} \mathrm{C}\end{array}$ & $\begin{array}{c}\Delta \mathbf{H}_{\mathrm{cc}} \\
\mathrm{J} / \mathrm{g}\end{array}$ & $\begin{array}{l}\mathrm{T}_{\mathrm{m}} \\
{ }^{\circ} \mathrm{C}\end{array}$ & $\begin{array}{c}\Delta \mathbf{H}_{\mathrm{m}} \\
\mathrm{J} / \mathrm{g}\end{array}$ \\
\hline PPTF & 375 & 402 & 36 & 0.175 & - & - & 183 & 44 & 36 & 0.323 & 120 & 36 & 183 & 37 \\
\hline PBTF & 391 & 410 & 24 & 0.272 & 87 & 28 & 148 & 28 & 24 & 0.291 & 88 & 27 & 148 & 27 \\
\hline PPeTF & 382 & 402 & 8 & 0.144 & - & - & $52 / 63$ & $19 / 6$ & 8 & 0.308 & - & - & - & - \\
\hline PHTF & 384 & 402 & 7 & 0.008 & - & - & $49 / 95$ & $10 / 23$ & 2 & 0.289 & - & - & - & - \\
\hline
\end{tabular}



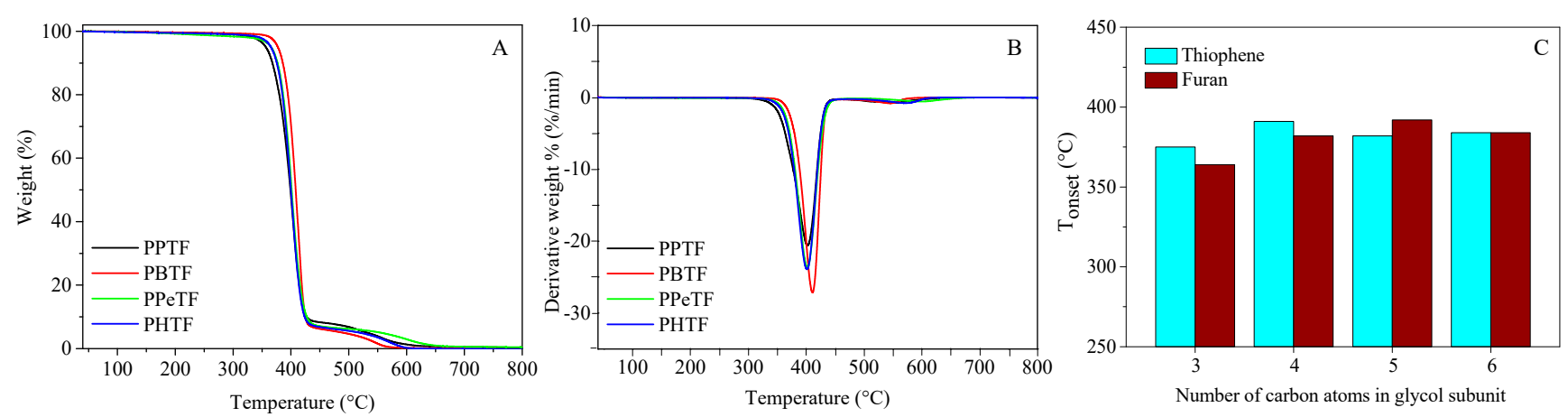

Figure 4. (A) TGA curves and (B) TGA derivatives of PPTF, PBTF, PPeTF and PHTF homopolymers; (C) comparison between $\mathrm{T}_{\text {onset }}$ of both thiophene- and furan-based homopolymers.

The highest thermal stability of PPeF within the furan-based family was ascribed to the presence in this polyester of the highest fraction of mesophase, the latter due to the establishment of hydrogen bonds between adjacent macromolecular chains in addition to $\pi-\pi$ interactions [33]. As previously reported, mesophase formation is favoured in flexible and mobile macromolecular chains and compete with the crystalline 3D phase. PPeF was indeed amorphous with $\mathrm{T}_{\mathrm{g}}$ below room temperature and had very slow crystallising capability $[29,33,34]$. The authors of the present paper previously investigated PBTF, which was characterised by the presence of a 2D-ordered phase (mesophase) $[16,18]$. Nevertheless, the mesophase present in thiophene-based polyesters cannot originate from hydrogen bonds between adjacent polymer chains, sulphur atoms being significantly less electronegative than oxygen. In this case, the $2 \mathrm{D}$-ordered structure could arise from thiophene ring $\pi-\pi$ stacking, as observed also for polythiophenes [35] and for some other aromatic polyesters containing, for example, terephthalic and isophthalic rings [36-38]. As previously reported $[16,18]$, also for the thiophene-based family, the mesophase formed at the expense of the crystalline one, particularly enhanced by compression-moulding treatment.

The DSC analysis discussed below indicates PBTF compression-moulded film is characterised by a very low crystallinity degree, different from the other members of the family. Consequently, we can hypothesize the mesophase fraction, and then the interchain $\pi-\pi$ stacking interactions, reached the maximum value in the PBTF film, determining the highest thermal degradation temperature.

The first scan DSC traces of compression-moulded films are shown in Figure 5 and the relative data are displayed in Table 2.
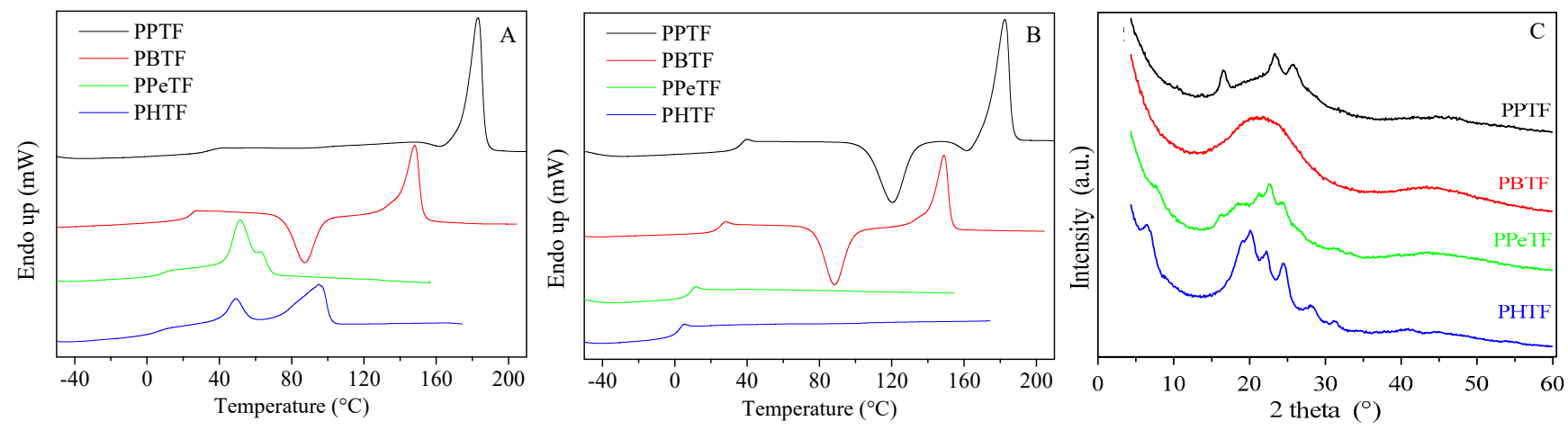

Figure 5. (A) I scan; (B) II scan DSC curves; (C) WAXS profiles of PPTF, PBTF, PPeTF and PHTF compression-moulded films.

First of all, it has to be emphasized that some remarkable differences in the thermal behaviour before and after compression moulding were noticed. In all cases, with the exception of PBTF film, the I scan curves show endotherms typical of semicrystalline materials. In the case of PBTF, an additional exothermic peak between $\mathrm{T}_{\mathrm{g}}$ and $\mathrm{T}_{\mathrm{m}}$ could be observed; however, the peak areas were comparable, $\left(\Delta \mathrm{H}_{\mathrm{cc}}=\Delta \mathrm{H}_{\mathrm{m}}\right)$, indicating film 
processing totally suppressed the development of 3D-crystalline phase in the PBTF sample. As for PPeTF, analogously to powder, a broad endothermic phenomenon could be found. However, in this case, the peak at the lower temperature $\left(52^{\circ} \mathrm{C}\right)$ was more intense than that at the higher temperature $\left(63^{\circ} \mathrm{C}\right)$. Finally, the DSC curve of PHTF film shows two melting peaks, at 49 and $95{ }^{\circ} \mathrm{C}$, very similar to the endotherms measured for purified powder, but again higher intensity for the lower temperature signal was detected. Interestingly, in both PPeTF and PHTF polyesters the first endothermic peak was located at a constant temperature, about $50{ }^{\circ} \mathrm{C}$. It is worth noticing the signal, similar in terms of position even if less intense, previously reported for PBTF film was ascribed to the mesophase isotropisation phenomenon [16]. Such an endotherm is not visible in the calorimetric trace of the PBTF film obtained from dimethyl 2,5-thiophenedicarboxylate [18], probably hidden by the upcoming cold crystallisation peak (as known, the mesophase can act as a precursor of the 3D crystalline phase). Considering this scenario, it can be hypothesized that the endotherms around $50{ }^{\circ} \mathrm{C}$ in both PPeTF and PHTF were due to this peculiar 2D microstructure already detected and investigated.

As expected, II scan DSC curves of films are identical to those collected for purified powders.

In Figure 5C, the WAXS profiles of films are reported. From the profile shape, we can state PPTF and PHTF were in the same crystal forms as the respective powders. Indeed, the peak positions of PPTF film were $16.5^{\circ}, 23.3^{\circ}$ and $25.5^{\circ}$, very similar to the powder ones; PHTF showed peaks at $6.5^{\circ}, 19.1^{\circ}, 20.0^{\circ}, 22.2^{\circ}, 24.4^{\circ}, 28.2^{\circ}$ and $31.2^{\circ}$. For this sample, the position of the peaks was not exactly the same as those observed in the powder sample, but the good crystallinity and the overall match of the pattern allow us to state the powder and the film displayed the same crystal form, the small differences observed being due to different preparations. PBTF film showed only the bell-shaped background, typical of amorphous compounds, in agreement with DSC results. The comparison of PPeTF samples was not easy, the film showing small peaks or shoulders at $7.5^{\circ}, 16.3^{\circ}, 18.4^{\circ}, 21.3^{\circ} 22.6^{\circ}$ and $24.3^{\circ}$. They were roughly at the same positions found in the powder sample. The imperfect coincidence in the position of the peaks as well as the presence of additional peaks could be compatible with the presence of a further phase.

As for sample crystallinity, $X_{c}$ values were $18 \%, 14 \%$ and $21 \%$ for PPTF, PPeTF and PHTF, respectively.

For the sake of comparison, in Figure $\mathrm{S} 3 \mathrm{~T}_{\mathrm{g}}$ and $\mathrm{T}_{\mathrm{m}}$ of the thiophene-based polyesters under study and their furan- and terephthalate-based counterparts are reported as a function of glycolic subunit length. As for $T_{g}$ values, regardless of the kind of ring considered, all the families showed a regular decrease of glass transition temperature with the glycolic subunit length. In addition, by fixing the glycol length, the trend observed was $\mathrm{T}_{\mathrm{g} \text {,thiophene }}<\mathrm{T}_{\mathrm{g} \text {,benzene }} \leq \mathrm{T}_{\mathrm{g} \text {,furan }}$, more pronounced for 1,3-propanediol- and 1,4-butanediol-containing polymers, as a result of the different polarity /aromaticity ratio of the three rings. The lower $\mathrm{T}_{\mathrm{g}}$ values of thiophene-based polyesters with respect to those of the furan-based ones can be explained considering that in the former polymer family interchain interactions are weaker because of the lower electronegativity of sulphur atoms with respect to oxygen ones.

As for the melting temperatures, although the same trend can be noticed for all the polymeric families, the decrease of $\mathrm{T}_{\mathrm{m}}$ for thiophene-based samples was shifted at higher $\mathrm{CH}_{2}$ numbers with respect to their furan- and benzene-based analogues, probably due to differences in the main structural parameters, which led to a less efficient chain packing, i.e., lower $T_{m} s$. PPTF and PPF were exceptions: the higher $T_{m}$ of the former polymer can be ascribed to a more perfect crystalline phase as well as to the higher aromaticity of thiophene ring, which promotes chain folding [15]. 


\subsection{Mechanical Characterisation}

Mechanical properties of the compression-moulded films were evaluated by tensile tests. The values of the elastic modulus $(\mathrm{E})$, stress at break $\left(\sigma_{\mathrm{B}}\right)$, and strain at break $\left(\varepsilon_{\mathrm{B}}\right)$ are displayed in Table 3 . The corresponding stress-strain curves are shown in Figure 6.

Table 3. Mechanical and gas permeability characterisation data of the compression-moulded films.

\begin{tabular}{|c|c|c|c|c|c|}
\hline & $\begin{array}{c}\mathrm{E} \\
(\mathrm{MPa})\end{array}$ & $\begin{array}{c}\sigma_{\mathrm{B}} \\
(\mathrm{MPa})\end{array}$ & $\begin{array}{c}\mathcal{E}_{B} \\
(\%)\end{array}$ & $\begin{array}{c}\mathrm{O}_{2} \text {-TR } \\
\left(\mathrm{cm}^{3} \mathrm{~cm} \mathrm{~m}^{-2} \mathrm{~d}^{-1} \mathrm{~atm}^{-1}\right)\end{array}$ & $\begin{array}{c}\mathrm{CO}_{2}-\mathrm{TR} \\
\left(\mathrm{cm}^{3} \mathrm{~cm} \mathrm{~m}^{-2} \mathrm{~d}^{-1} \mathrm{~atm}^{-1}\right)\end{array}$ \\
\hline PPTF & $1550 \pm 125$ & $13 \pm 3$ & $1.2 \pm 0.3$ & $0.021 \pm 0.003$ & $0.024 \pm 0.002$ \\
\hline PBTF & $47 \pm 3$ & $19 \pm 2$ & $580 \pm 60$ & $0.003 \pm 0.001$ & $0.017 \pm 0.001$ \\
\hline PPeTF & $\begin{array}{l}1.9 \pm 0.3^{*} \\
391 \pm 26\end{array}$ & $\begin{array}{l}0.5 \pm 0.1 * \\
14 \pm 2\end{array}$ & $\begin{array}{c}1650 \pm 110^{*} \\
17 \pm 3\end{array}$ & $0.288 \pm 0.035$ & $0.753 \pm 0.125$ \\
\hline PHTF & $\begin{array}{l}3.5 \pm 0.5^{*} \\
299 \pm 22\end{array}$ & $\begin{array}{l}0.5 \pm 0.1 * \\
15 \pm 1\end{array}$ & $\begin{array}{c}674 \pm 9^{*} \\
32 \pm 3\end{array}$ & $0.404 \pm 0.101$ & $1.62 \pm 0.110$ \\
\hline
\end{tabular}

${ }^{*}$ Mechanical data obtained immediately after film moulding.

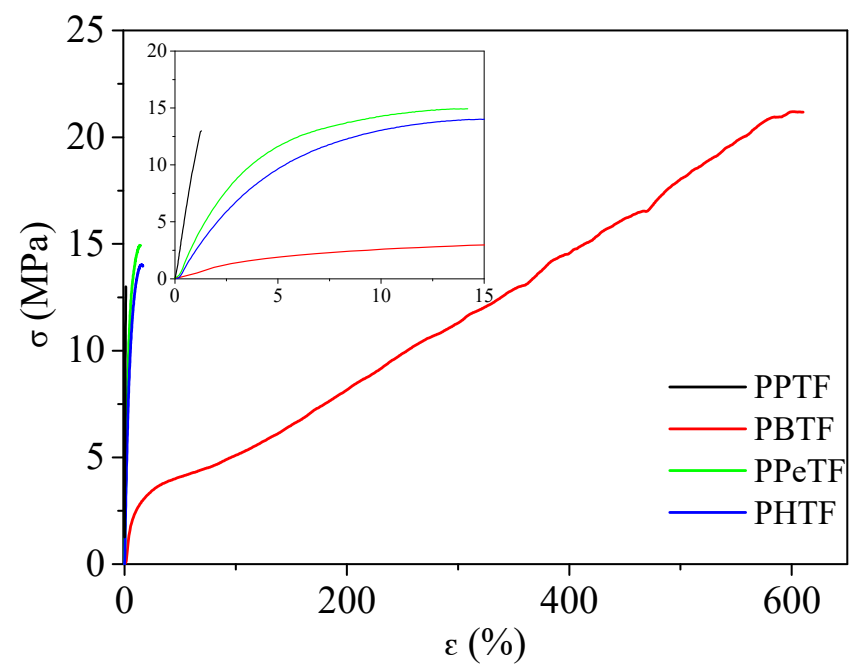

Figure 6. Stress-strain curves of PPTF, PBTF, PPeTF and PHTF films after 3 weeks of storage at room temperature.

The results obtained can be explained on the basis of chain flexibility (i.e., $\mathrm{T}_{\mathrm{g}}$ value) and crystallinity degree, which are the main parameters affecting polymers' mechanical response. More in detail, referring to mechanical characterisation data obtained after three weeks of storage at room temperature, it was possible to notice that PPTF was the most rigid material, with the highest value of $\mathrm{E}$ and the lowest elongation at break. This result was due both to its crystallinity degree, the highest among the family, and its $\mathrm{T}_{\mathrm{g}}$, the latter being well-above room temperature, which limited macromolecular mobility. Among the thiophene-based family, PBTF, which was amorphous with $\mathrm{T}_{\mathrm{g}}$ around RT, turned out to be the one with the lowest value of $\mathrm{E}$ and the highest of $\varepsilon_{\mathrm{B}}$, almost $600 \%$. Regarding polymers containing longer and flexible glycols, i.e., PPeTF and PHTF, which are both semicrystalline and in the rubbery state at RT, they were characterised by a mechanical response intermediate between those of PPTF and PBTF. The elongation at break was similar for the two polymers, whereas PPeTF exhibited a higher elastic modulus despite its lower crystallinity degree probably because of its lower chain flexibility (higher $\mathrm{T}_{\mathrm{g}}$ ). Lastly, for all the materials analysed, stress at break was comparable, with values ranging from 13 to $19 \mathrm{MPa}$.

The unexpected mechanical response of PTBF can be explained as being due to the presence of a mesophase in the polymeric film, as already demonstrated in previous studies carried out by some of the authors of the present paper [16,18]. As reported in the literature, 
polymer liquid crystals are characterised by smart mechanical properties, their brittleness being significantly reduced (high values of elongation at break).

In order to support the already-mentioned hypothesis of the presence of a mesophase, stress-strain measurements were carried out on PPeTF and PHTF films immediately after compression moulding, as reported in Figure 7. In these conditions, the two films were completely amorphous, as evidenced by DSC (only the endothermic baseline deviation associated with glass transition was evident in the DSC trace of both polyesters) and WAXS analysis (WAXS profiles of both polymers show only the bell shape halo characteristic of the amorphous phase) (Figure 7B,C,E,F). Nevertheless, it is worth noting that although both materials were in an amorphous rubbery state at room temperature, free-standing films could be obtained. That supports the assumption of the presence, together with the amorphous fraction, of another phase. A similar behaviour was already evidenced for PPeF, the furan-based analogous of PPeTF [29,33], for which the presence of a mesophase was demonstrated. As one can see from Figure 7, the mechanical response of the films tested immediately after moulding was completely different. Both films were characterised by outstanding elongation at break (around $1700 \%$ in the case of PPeTF and about $700 \%$ for PHTF) and elastic modulus values, about two orders of magnitude lower than those of the same films measured after 3 weeks from moulding (Figure 7A,D). This result further supposes the development of a mesophase in PPeTF and PHTF, too. The storage of the films at room temperature also favoured, of course, the formation of a 3D-crystalline phase. The former is associated with the endothermic phenomenon at constant $\mathrm{T}$ around $50{ }^{\circ} \mathrm{C}$, the latter to the fusion process occurring at a higher temperature whose value increases with the number of methylene groups present in the glycol subunit (see Figure 7B,E). Both the films become more rigid ( $\mathrm{E}$ increases) and stretch much less after 3 weeks at room temperature due to the increment of crystalline fractions and to disclinations between amorphous, 2D and 3D ordered phases.
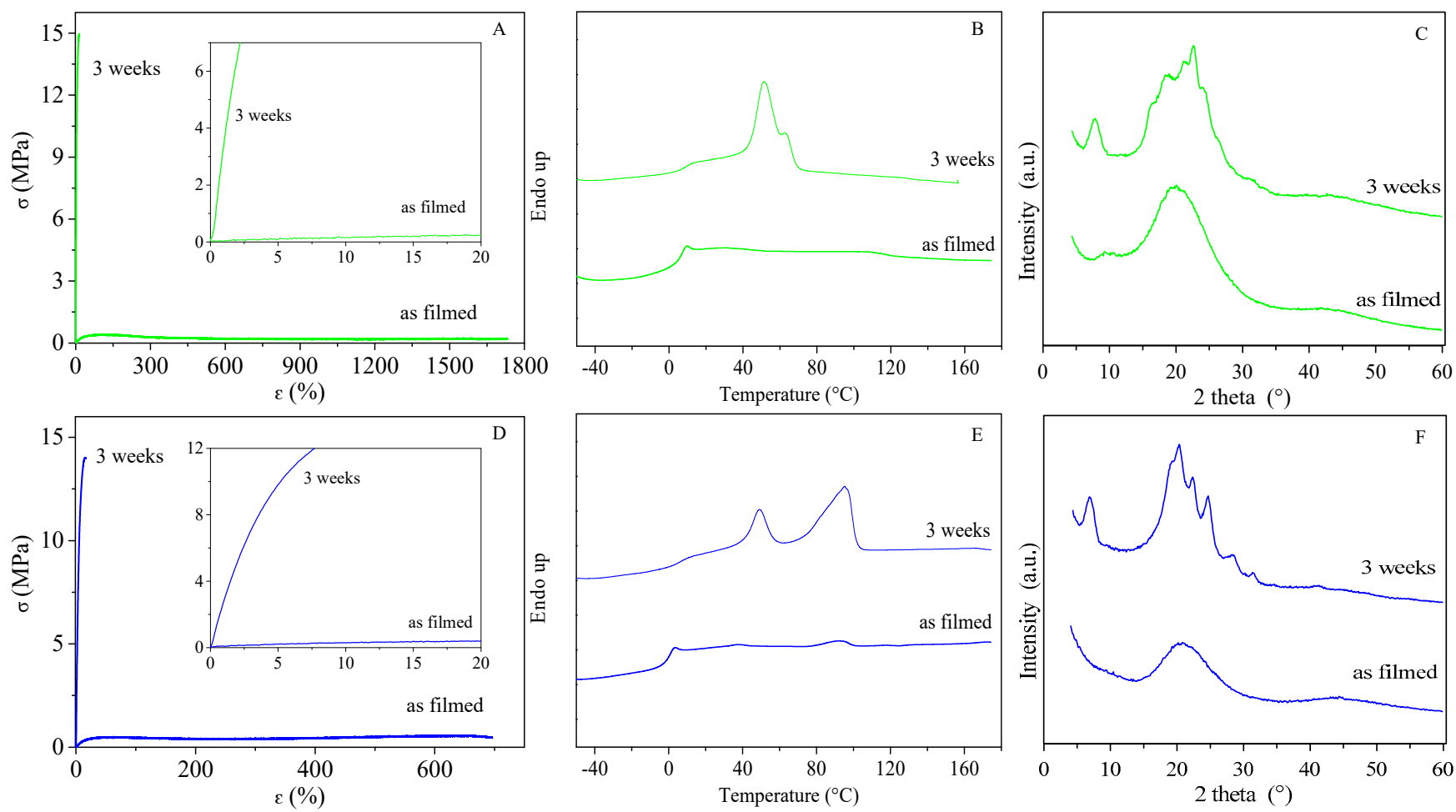

Figure 7. (A) Stress-strain curves; (B) DSC curves; (C) WAXS profiles of PPeTF films immediately after moulding and after 3 weeks of storage at room temperature. (D) Stress-strain curves; (E) DSC curves; (F) WAXS profiles of PHTF films immediately after moulding and after 3 weeks of storage at room temperature. 
In Figure 8, a comparison between mechanical characterisation data of thiophenebased homopolymers and their furan-based counterparts is shown. As far as the elastic modulus is concerned, an odd-even - $\mathrm{CH}_{2}$ - number trend was observed: the thiophenebased homopolymers containing a glycol subunit with an odd number of $-\mathrm{CH}_{2}$ - groups (PPTF and PPeTF) showed a higher elastic modulus with respect to their furan-based counterparts, attributable to their semicrystalline nature. An opposite result was found for the samples containing an even number of $\mathrm{C}$ atoms in glycolic subunits (PBTF vs. PBF and PHTF vs. PHF); in fact, the thiophene-based polyesters showed a lower elastic modulus than the furan-based analogous. More in detail, PBF film was semicrystalline, whereas PBTF was fully amorphous and PHF had a higher fraction of crystalline phase compared to PHTF. Moreover, the PHF crystal phase was characterised by a higher melting temperature with respect to PHTF, indicating a higher degree of perfection, i.e., the crystal phase was highly packed.
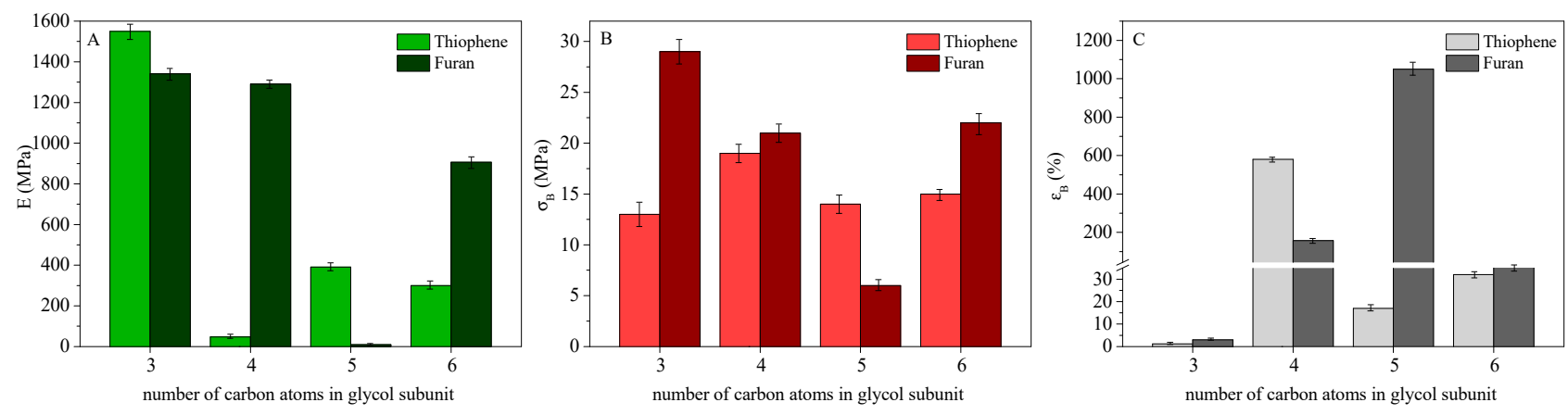

Figure 8. Comparison between mechanical characterisation data of thiophene-based homopolymers and their furan-based counterparts: (A) elastic modulus; (B) stress at break; (C) elongation at break.

In the case of stress at break, we measured in general a lower value for thiophenebased polyesters when compared with their furan-based counterparts, with the exception of polyesters of 1,5 pentanediol. For PPeTF and PPeF, exactly the opposite occurred. Interestingly, $\sigma_{\mathrm{B}, \mathrm{PPTF}}<\sigma_{\mathrm{B}, \mathrm{PPF}}$, despite the semicrystalline nature of the former film and the amorphous nature of the latter one. Such a result can be correlated with the significantly higher $\mathrm{T}_{\mathrm{g}}$ of PPF. For the pair PBTF and PBF, the lower $\sigma \mathrm{B}$ of the thiophene-based polyester was due to its amorphous nature, $\mathrm{PBF}$ being on the contrary semicrystalline. As far as PPeTF and PPeF are concerned, $\sigma_{\mathrm{B}, \mathrm{PPeTF}}>\sigma_{\mathrm{B}, \mathrm{PPeF}}$ because of the semicrystalline nature of thiophene-containing film and the amorphous character of the furan-based one. Lastly, PHF film is characterised by a higher stress at break than the PHTF one due to its higher crystalline degree, $T_{m}$ and $T_{g}$ values.

The elongation at break was also correlated with the presence in the film of a crystalline phase; in fact, $\varepsilon_{\mathrm{B}, \mathrm{PBTF}}>\varepsilon_{\mathrm{B}, \mathrm{PBF}}$, (PBTF film was amorphous, while PBF contained a crystal phase). Exactly the opposite happened for the PPeTF and PPeF pair; the former was indeed semicrystalline and the latter was amorphous. Lastly, PHTF and PHF were characterised by a very similar elongation at break, as expected if we considered both polyesters are semicrystalline and in the rubbery state.

\subsection{Gas Barrier Properties Evaluation}

Barrier properties were evaluated with respect to dry $\mathrm{O}_{2}$ and $\mathrm{CO}_{2}$ gases. The GTR values normalised for the sample thickness and measured at $23^{\circ} \mathrm{C}$ and at $0 \%$ of relative humidity are displayed in Table 3. For the sake of comparison, in Figure 9A,B gas permeability values of thiophene-based materials are shown together with those of their furan-based counterparts and with some commonly used traditional fossil-based plastics and other bio-based polymers, respectively.

As is well-known, many factors, such as ordered and amorphous phases amount, glass transition temperature, chain polarity and flexibility, as well as molecular weight 
and its distribution, can affect the final barrier properties of materials together with the different characteristics of gas molecules (like size, polarity, inertness). Since gases cannot diffuse and permeate through the highly packed crystalline phase, this parameter plays a key role. Therefore, it is generally assumed that in polymers with higher percentages of crystalline phases the best barrier performances are observed. In addition, it is wellrecognized the glassy state offers a higher barrier to gases since it is characterised by reduced chain mobility with respect to the rubbery one, and also by a lower free volume through which gas can diffuse. When mesogenic groups, like thiophene rings, are present together with flexible segments, barrier properties can further improve thanks to the development of a mesophase (1D- or 2D-ordered structure), as this is even more effective than the crystalline phase in hampering gas passage [39]. As already mentioned, mesoand crystalline phases develop one at the expense of the other. Moreover, when both form the amorphous-mesophase-crystal disclination content raises. According to these assumptions, it is not surprising PBTF turned out to be the best-performing material of the family, being the sample in which just the most performant mesophase was present together with the disordered portion, i.e., less interphase percentage.

In addition, previous studies carried out on poly(butylene isophthalate) PBI, which similar to PBTF is aromatic with a $\mathrm{T}_{\mathrm{g}}$ near $\mathrm{T}_{\text {room }}$, have shown this material, if stored at room temperature, develops a very efficiently packed amorphous phase within a few minutes $[40,41]$. The presence of this peculiar dense amorphous phase can explain the excellent mechanical and barrier properties of PBI. Conversely, in poly(ethylene terephthalate) (PET), another well-known aromatic polyester, a very different behaviour was observed: being PET $\mathrm{T}_{\mathrm{g}}$ is well-above room temperature, it is not capable of quick re-arrangement, and the above-mentioned densification does not occur in short times. All these studies then seem to support the assumption according to which the mesophase presence and the possibility of quickly compacting its mobile amorphous phase are responsible for the outstanding PBTF properties. According to the data reported in Table 3, PPTF is the second-best performing material: it is indeed the only one that showed a $T_{g}$ value above room temperature, which means an amorphous glassy phase characterised by a low amount of free volume through which gases can diffuse. As for PPeTF and PHTF, both contained a rubbery amorphous phase at room temperature, coexisting with a mesophase and crystals. Consequently, their higher GTR values can be explained on the basis of the higher fraction of free volume due to $\mathrm{T}_{\mathrm{g}}>\mathrm{RT}$ and to the contemporary presence of both $3 \mathrm{D}$ and $2 \mathrm{D}$ domains causing higher disclination (channels created at the interface between the two ordered regions) density through which gas can easily diffuse. Conversely, there was no significant separation between the mesophase and amorphous regions, as they were characterised by a similar electron density.

Moreover, for all the samples under study, $\mathrm{CO}_{2}$ was more permeable than $\mathrm{O}_{2}$, in agreement with studies carried out in the literature on other similar polymeric systems $[42,43]$ due to reduction of diffusivity with the decreasing of the permeant size (the values of molecular diameter for $\mathrm{CO}_{2}$ and $\mathrm{O}_{2}$ were $3.4 \AA$ and $3.1 \AA$, respectively) [44].

The $\mathrm{GTR}_{\mathrm{CO} 2} / \mathrm{GTR}_{\mathrm{O} 2}$ ratio changed as a function of glycol subunit length; for PPTF (containing 3 methylene groups) it was close to 1, turning almost 2 in the case of PBTF, and 2.5 for PPeTF and PHTF (containing 5 and 6 methylene moieties, respectively). $\mathrm{GTR}_{\mathrm{CO} 2} / \mathrm{GTR}_{\mathrm{O} 2}$ ratio increments can be associated with a decrement of $\mathrm{CO}_{2}$ solubility in the polymer matrix occurring in samples containing longer glycol subunits.

If thiophene-based homopolymers are compared to their furan-based counterparts, some significant differences can be detected. First, within the thiophene family, the bestperforming material was the one containing four carbon atoms in the glycol subunit, unlike what was found for the furan family where PPeF resulted in the best one $[29,33]$. It is worth noting both the samples were totally amorphous and with a $\mathrm{T}_{\mathrm{g}}$ around RT; it can be supposed that this latter condition favoured the formation of a mesophase. It seems the optimal glycolic length to maximize mesophase formation was different for the two classes, probably because the two mesophases arose from different intermolecular interactions 
( $\pi-\pi$ stacking in the case of poly(alkylene $2,5-$ thiophenedicarboxylate)s, $\pi-\pi$ stacking and intermolecular hydrogen bonds for poly(alkylene 2,5-furandicarboxylate)s).
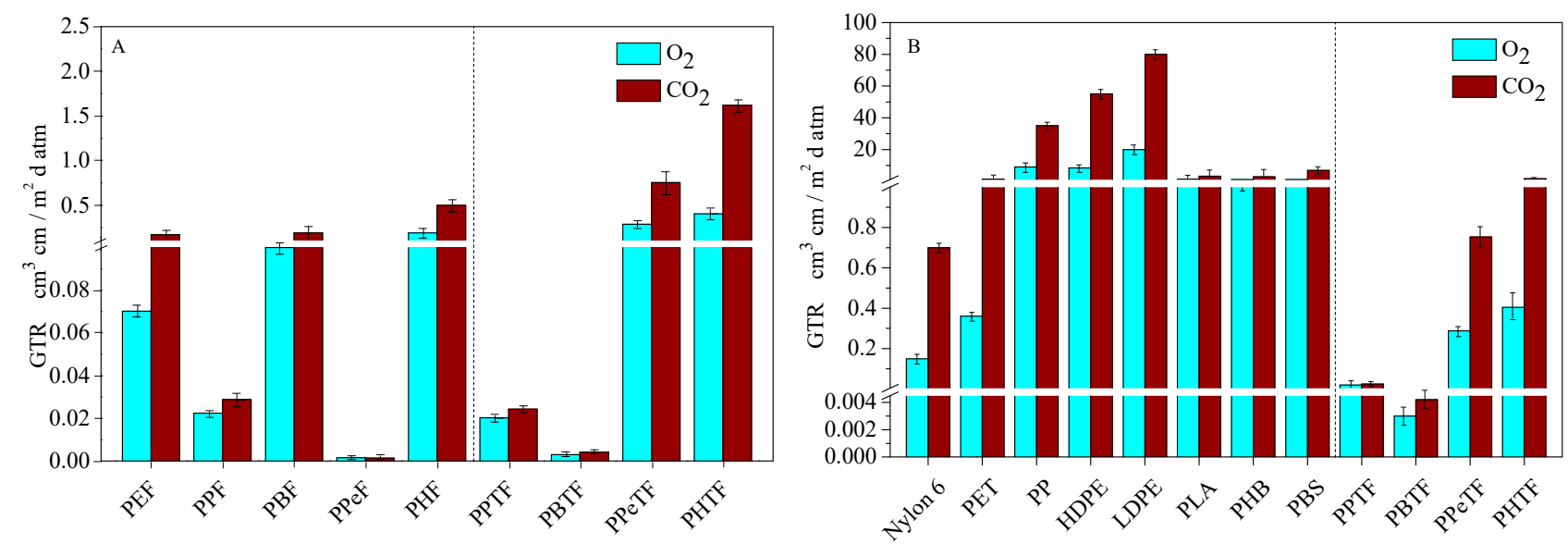

Figure 9. GTR values of $\mathrm{O}_{2}$ and $\mathrm{CO}_{2}$ through polymeric films $\left(T=23{ }^{\circ} \mathrm{C}\right)$ compared with those of (A) furan-based homopolymers [29,33]; (B) traditional plastics and principal bio-based polymers [8,18,45].

In addition, by comparing furan- and thiophene-based homopolymers containing a propylene segment, it can be noticed that $\mathrm{GTR}_{\mathrm{PPTF}}<\mathrm{GTR}_{\mathrm{PPF}}$. Both were in the glassy state, but the former was semicrystalline, the latter amorphous. Mesophase formation was not favoured in both cases, the macromolecular chains being frozen in the glassy state. Considering the butylene moiety-containing polyesters, the results recorded evidence PBTF was more performant than PBF. That was probably due to the presence of a higher amount of mesophase, whose formation was favoured by an amorphous and partially mobile phase ( $\mathrm{PBF}$, on the contrary, was glassy and semicrystalline). The five methylene-containing polymers, $\mathrm{PPeF}$ and PPeTF, were both in the rubbery state at room temperature, with PPeTF containing also a crystal phase. Nevertheless, GTR $\mathrm{GPeF}<<<\mathrm{GTR}_{\mathrm{PPeTF}}$ due to the very high mesophase fraction in the PPeF sample. Lastly, GTR $R_{P H F}<G_{\text {THTF }}$ is explainable on the basis of a higher $\mathrm{T}_{\mathrm{g}}$ and crystalline fraction for PHF.

Looking at the overall trend, by lengthening the glycol subunit in the poly(alkylene 2,5-thiophenedicarboxylate)s, we can observe a progressive worsening of barrier properties, except for PBTF due to the lowering of $\mathrm{T}_{\mathrm{g}}$ values, unlike furan-based polyesters for which an even-odd trend was observed [29]. It can be assumed that for PPeTF and PHTF, despite the higher amount of mesophase, the worsening effect of both low $\mathrm{T}_{\mathrm{g}}$ value and the high amount of disclinations between the mesophase and 3D domains prevailed.

The homopolymers under study were also compared to the fossil-based traditional materials currently employed in food packaging, as well as to other bio-based polyesters (see Figure 9B). Poly(alkylene 2,5-thiophenedicarboxylate)s turned out to be the more performant of the polyolefins and of PLA, PHB and PBS to both gases. Interestingly, PPTF and PBTF had even better barrier properties than PET and nylon.

\section{Conclusions}

A new family of fully bio-based homopolymers of 2,5-thiophenedicarboxylic acid were successfully synthesized via melt polycondensation, a green and solvent-free process, starting from dimethyl 2,5-thiophenedicarboxylate and glycols with different lengths.

All solid-state properties appeared to be strongly affected by glycol subunit length; the number of methylene groups present in the flexible aliphatic segment indeed impacted the macromolecular chain flexibility and crystallising ability, in turn, determining different glass transition temperature values and the development of different kinds and fractions of ordered phases (2D mesophase or 3D crystalline phase).

Through the analysis of the effect of glycol subunit length on sample microstructure, we could confirm some results previously reported: 
- Mesophase formation is favoured in the case of macromolecular chains, which are mobile at room temperature but have reduced crystallising capability. If the glycol subunit is long enough, 3D crystalline phase formation appears prevalent

- Mesophases and crystalline phases compete with each other, i.e., each one develops at the expense of the other

- Type, number, and amount of ordered phases have a huge impact on the final functional properties (mechanical and gas barrier)

As previously established for furan-based polyesters, the outstanding gas barrier properties are mainly ascribable to mesophase presence. However, due to the lower electronegativity of sulphur atoms with respect to oxygen ones, the 2D-ordered structure present in poly(alkylene 2,5-thiophenedicarboxylate)s could only originate from thiophene ring $\pi-\pi$ stacking, different from furan-based polyesters where the mesophase also resulted from interchain hydrogen bonds involving furan rings.

This is the reason why the result of the comparison between the functional properties of a thiophene-based polyester and the furan-based analogue with the same length of the glycol subunit changed according to the length of this latter.

Of particular importance is the comparison in terms of functional properties between the best-performing polymer of the thiophene-based family, namely PBTF, and PPeF, the best polymer within the family of 2,5-furandicarboxylic acid. Both polyesters were characterised by outstanding barrier properties, even though some differences, directly related to the nature of interchain interactions responsible of the resulting mesophase, exist: the strong interchain hydrogen bonds established in PPeF in addition to van der Waals $\pi-\pi$ interactions, also present among thiophene rings, explain the halving of the oxygen GTR value observed for the PPeF, whereas the lower dipole moment of the thiophene ring with respect to the furan one justified the doubling of the permselectivity ratio in the case of PBTF. In both cases, we are dealing with polymers with performances similar to EVOH, which is used as a high gas barrier material in multilayer films.

On the other hand, PBTF is more tough and ductile than PPeF, having an elastic modulus and a stress at break 5 and 3 times higher, respectively, than those of PPeF, the elongation at break being very high $(>500 \%)$.

Supplementary Materials: The following are available online at https:/ /www.mdpi.com/article/ 10.3390/polym13152460/s1, Figure S1: Pictures of compression-moulded films, Figure S2: Chromatograms of the synthesised polymers, Table S1: DSC data of the homopolymers in the form of purified powders, Figure S3: $\mathrm{T}_{\mathrm{g}}$ and $\mathrm{T}_{\mathrm{m}}$ trends as a function of glycolic subunit length for thiophene ring-containing polyesters compared to furan ring- and benzene ring-containing ones.

Author Contributions: Conceptualisation, M.S. and N.L.; methodology, G.G., V.S. and M.G.; investigation, G.G., V.S., M.S., M.G. and N.L.; data curation, G.G., V.S., M.S., M.G. and N.L.; writingoriginal draft preparation, G.G. and N.L.; writing—review and editing, G.G., M.S. and N.L.; supervision, M.S. and N.L. All authors have read and agreed to the published version of the manuscript.

Funding: This research received no external funding.

Institutional Review Board Statement: Not applicable.

Informed Consent Statement: Not applicable.

Data Availability Statement: The data that support the findings of this study are available from the corresponding author upon reasonable request.

Conflicts of Interest: The authors declare no conflict of interest.

\section{References}

1. Plastics Europe-Plastics the Facts. 2020. Available online: https:/ /www.plasticseurope.org (accessed on 12 June 2021).

2. European Bioplastics-Bioplastics Market Data. 2020. Available online: https://docs.european-bioplastics.org (accessed on 12 June 2021).

3. European Commission-A European Strategy for Plastics in a Circular Economy. 2015. Available online: https:/ / ec.europa.eu/ environment/circular-economy/pdf/plastics-strategy-brochure.pdf (accessed on 12 June 2021). 
4. Top Value Added Chemicals from Biomass Volume I-Results of Screening for Potential Candidates from Sugars and Synthesis Gas. 2004. Available online: https:/ / www.nrel.gov/docs/fy04osti/35523.pdf (accessed on 12 June 2021).

5. $\quad$ Burgess, S.K.; Leisen, J.E.; Kraftschik, B.E.; Mubarak, C.R.; Kriegel, R.M.; Koros, W.J. Chain Mobility, Thermal, and Mechanical Properties of Poly(ethylene furanoate) Compared to Poly(ethylene terephthalate). Macromolecules 2014, 47, 1383-1391. [CrossRef]

6. $\quad$ Burgess, S.K.; Mikkilineni, D.S.; Yu, D.B.; Kim, D.J.; Mubarak, C.R.; Kriegel, R.M.; Koros, W.J. Water sorption in poly(ethylene furanoate) compared to poly(ethylene terephthalate). Part 1: Equilibrium sorption. Polymer 2014, 55, 6861-6869. [CrossRef]

7. $\quad$ Burgess, S.K.; Mikkilineni, D.S.; Yu, D.B.; Kim, D.J.; Mubarak, C.R.; Kriegel, R.M.; Koros, W.J. Water sorption in poly(ethylene furanoate) compared to poly(ethylene terephthalate). Part 2: Kinetic sorption. Polymer 2014, 55, 6870-6882. [CrossRef]

8. Burgess, S.K.; Kriegel, R.M.; Koros, W.J. Carbon Dioxide Sorption and Transport in Amorphous Poly(ethylene furanoate). Macromolecules 2015, 48, 2184-2193. [CrossRef]

9. Wang, J.; Liu, X.; Jia, Z.; Liu, Y.; Sun, L.; Zhu, J. Synthesis of bio-based poly(ethylene 2,5-furandicarboxylate) copolyesters: Higher glass transition temperature, better transparency, and good barrier properties. J. Polym. Sci. Part A Polym. Chem. 2017, 55, 3298-3307. [CrossRef]

10. Van Putten, R.J.; Van der Waal, J.C.; De Jong, E.; Rasrendra, C.B.; Heeres, H.J.; De Vries, J.G. Hydroxymethylfurfural, A Versatile Platform Chemical Made from Renewable Resources. Chem. Rev. 2013, 113, 1499-1597. [CrossRef]

11. Zhi, W.; Hu, Y.; Liang, M.; Liu, Y.; Li, J.; Yin, J.; Shi, Y. Solid-liquid equilibrium and thermodynamic of 2,5-thiophenedicarboxylic acid in different organic solvents. Fluid Phase Equilib. 2014, 375, 110-114. [CrossRef]

12. Yang, Y.; Zhang, Q.; Cao, C.; Cheng, L.; Shi, Y.; Yang, W.; Hu, Y. Solubility and solution thermodynamics of 2,5thiophenedicarboxylic acid in (water + ethanol) binary solvent mixtures. Thermochim. Acta 2014, 592, 52-57. [CrossRef]

13. Polen, T.; Spelberg, M.; Bott, M. Toward biotechnological production of adipic acid and precursors from biorenewables. J. Biotechnol. 2013, 167, 75-84. [CrossRef]

14. Corona, A.; Biddy, M.J.; Vardon, D.R.; Birkved, M.; Hauschild, M.Z.; Beckham, G.T. Life cycle assessment of adipic acid production from lignin. Green Chem. 2018, 20, 3857-3866. [CrossRef]

15. Guidotti, G.; Soccio, M.; Lotti, N.; Gazzano, M.; Siracusa, V.; Munari, A. Poly(propylene 2,5-thiophenedicarboxylate) vs. poly(propylene 2,5-furandicarboxylate): Two examples of high gas barrier bio-based polyesters. Polymers 2018, 10, 785. [CrossRef] [PubMed]

16. Guidotti, G.; Gigli, M.; Soccio, M.; Lotti, N.; Gazzano, M.; Siracusa, V.; Munari, A. Poly(butylene 2,5-thiophenedicarboxylate): An added value to the class of high gas barrier biopolyesters. Polymers 2018, 10, 167. [CrossRef] [PubMed]

17. Guidotti, G.; Gigli, M.; Soccio, M.; Lotti, N.; Salatelli, E.; Gazzano, M.; Siracusa, V.; Munari, A. Tailoring poly(butylene 2,5thiophenedicarboxylate) features by the introduction of adipic acid co-units: Biobased and biodegradable aliphatic/aromatic polyesters. Polymer 2018, 145, 11-20. [CrossRef]

18. Guidotti, G.; Gigli, M.; Soccio, M.; Lotti, N.; Gazzano, M.; Siracusa, V.; Munari, A. Ordered structures of poly(butylene 2,5thiophenedicarboxylate) and their impact on material functional properties. Eur. Polym. J. 2018, 106, 284-290. [CrossRef]

19. Wang, G.Q.; Liang, Y.; Jiang, M.; Zhang, Q.; Wang, R.; Wang, H.H.; Zhou, G.Y. Synthesis and characterization of bio-based polyesters from 2,5-thiophenedicarboxylic acid. Polym. Degrad. Stab. 2019, 168, 108942. [CrossRef]

20. Wang, G.Q.; Jiang, M.; Zhang, Q.; Wang, R.; Liang, Q.D.; Wang, H.H.; Zhou, G.Y. Partially bio-based and tough polyesters, poly(ethylene 2,5-thiophenedicarboxylate-co-1,4-cyclohexanedimethylene 2,5-thiophenedicarboxylate)s. Express Polym. Lett. 2019, 13, 938-947. [CrossRef]

21. Wang, G.Q.; Liang, Y.; Jiang, M.; Zhang, Q.; Wang, R.; Wang, H.H.; Zhou, G.Y. High Tg and tough poly(butylene 2,5thiophenedicarboxylate-co-1,4-cyclohexanedimethylene 2,5-thiophenedicarboxylate)s: Synthesis and characterization. J. Appl. Polym. Sci. 2020, 137, 48634. [CrossRef]

22. Wang, G.Q.; Jiang, M.; Zhang, Q.; Wang, R.; Liang, Q.D.; Zhou, G.Y. New bio-based copolyesters poly(trimethylene 2,5thiophenedicarboxylate-co-trimethylene terephthalate): Synthesis, crystallization behavior, thermal and mechanical properties. Polymer 2019, 173, 27-33. [CrossRef]

23. Wang, G.Q.; Jiang, M.; Zhang, Q.; Wang, R.; Liang, Q.D.; Zhou, G.Y. New bio-based copolyesters derived from 1,4-butanediol, terephthalic acid and 2,5-thiophenedicarboxylic acid: Synthesis, crystallization behavior, thermal and mechanical properties. Polym. Test. 2019, 75, 213-219. [CrossRef]

24. Wang, J.G.; Zhang, X.Q.; Shen, A.; Zhu, J.; Song, P.A.; Wang, H.; Liu, X.Q. Synthesis and Properties Investigation of Thiophenearomatic Polyesters: Potential Alternatives for the 2,5-Furandicarboxylic Acid-based Ones. Chin. J. Polym. Sci. 2020, 38, 1082-1091. [CrossRef]

25. Ferrario, V.; Pellis, A.; Cespugli, M.; Guebitz, G.M.; Gardossi, L. Nature Inspired Solutions for Polymers: Will Cutinase Enzymes Make Polyesters and Polyamides Greener? Catalysts 2017, 6, 205. [CrossRef]

26. Pellis, A.; Gamerith, C.; Ghazaryan, G.; Aortner, A.; Herrero Acero, E.; Guebitz, G.M. Ultrasound-enhanced enzymatic hydrolysis of poly(ethylene terephthalate). Bioresour. Technol. 2016, 218, 1298-1302. [CrossRef]

27. Weinberger, S.; Canadell, J.; Quartinello, F.; Yeniad, B.; Arias, A.; Pellis, A.; Guebitz, G.M. Enzymatic Degradation of Poly(ethylene 2,5-furanoate) Powders and Amorphous Films. Catalysts 2017, 7, 318. [CrossRef]

28. Gigli, M.; Quartinello, F.; Soccio, M.; Pellis, A.; Lotti, N.; Guebitz, G.M.; Licoccia, S.; Munari, A. Enzymatic hydrolysis of poly(1,4-butylene 2,5-thiophenedicarboxylate) (PBTF) and poly(1,4-butylene 2,5-furandicarboxylate) (PBF) films: A comparison of mechanisms. Environ. Int. 2019, 130, 104852. [CrossRef] [PubMed] 
29. Guidotti, G.; Soccio, M.; García-Gutiérrez, M.C.; Ezquerra, T.; Siracusa, V.; Gutiérrez-Fernández, E.; Munari, A.; Lotti, N. Fully Biobased Superpolymers of 2,5-Furandicarboxylic Acid with Different Functional Properties: From Rigid to Flexible, High Performant Packaging Materials. ACS Sustain. Chem. Eng. 2020, 8, 9558-9568. [CrossRef] [PubMed]

30. Dobbertin, J.; Hensel, A.; Schick, C. Dielectric spectroscopy and calorimetry in the glass transition region of semi-crystalline poly(ethylene terephthalate). J. Therm. Anal. 1996, 47, 1027-1040. [CrossRef]

31. Sanz, A.; Nogales, A.; Ezquerra, T.A.; Lotti, N.; Munari, A.; Funari, S.S. Order and segmental mobility during polymer crystallization: Poly(butylene isophthalate). Polymer 2006, 47, 1281-1290. [CrossRef]

32. Soccio, M.; Lotti, N.; Finelli, L.; Munari, A. Thermal characterization of novel aliphatic polyesters with ether and thioether linkages. e-Polymers 2009, 10, 35.

33. Guidotti, G.; Soccio, M.; García-Gutiérrez, M.C.; Gutiérrez-Fernández, E.; Ezquerra, T.A.; Siracusa, V.; Munari, A.; Lotti, N. Evidence of a 2D-Ordered Structure in Biobased Poly(pentamethylene furanoate) Responsible for Its Outstanding Barrier and Mechanical Properties. ACS Sustain. Chem. Eng. 2019, 7, 17863-17871. [CrossRef]

34. Martínez-Tong, D.E.; Soccio, M.; Robles-Hernández, B.; Guidotti, G.; Gazzano, M.; Lotti, N.; Alegria, A. Evidence of Nanostructure Development from the Molecular Dynamics of Poly(pentamethylene 2,5-furanoate). Macromolecules 2020, 53, 10526-10537. [CrossRef]

35. Rudyak, V.Y.; Gavrilov, A.A.; Guseva, D.V.; Tung, S.H.; Komarov, P.V. Accounting for $\pi-\pi$ stacking interactions in the mesoscopic models of conjugated polymers. Mol. Syst. Des. Eng. 2020, 5, 1137. [CrossRef]

36. Sago, T.; Itagaki, H.; Asano, T. Onset of Forming Ordering in Uniaxially Stretched Poly(ethylene terephthalate) Films Due to $\pi-\pi$ Interaction Clarified by the Fluorescence. Macromolecules 2014, 47, 217-226. [CrossRef]

37. Shanavas, A.; Sathiyaraj, S.; Chandramohan, A.; Narasimhaswamy, T.; Sultan Nasar, A. Isophthalic acid based mesogenic dimers: Synthesis and structural effects on mesophase properties. J. Mol. Struct. 2013, 1038, 126-133. [CrossRef]

38. Mahendrasingam, A.; Blundell, D.J.; Martin, C.; Urban, V.; Narayanan, T.; Fuller, W. Time resolved WAXS study of the role of mesophase in oriented crystallisation of poly(ethylene terephthalate-co-isophthalate) copolymers. Polymer 2005, 46, 6044-6049. [CrossRef]

39. Hedenqvist, M.S. Barrier Packaging Materials. In Handbook of Environmental Degradation of Materials, 2nd ed.; Kutz, M., Ed.; Elsevier, Inc.: Amsterdam, The Netherlands, 2012; pp. 840-842.

40. Quattrosoldi, S.; Lotti, N.; Soccio, M.; Schick, C.; Androsch, R. Stability of Crystal Nuclei of Poly (butylene isophthalate) Formed Near the Glass Transition Temperature. Polymers 2020, 12, 1099. [CrossRef] [PubMed]

41. Quattrosoldi, S.; Androsch, R.; Janke, A.; Soccio, M.; Lotti, N. Enthalpy Relaxation, Crystal Nucleation and Crystal Growth of Biobased Poly(butylene Isophthalate). Polymers 2020, 12, 235. [CrossRef]

42. Genovese, L.; Gigli, M.; Lotti, N.; Gazzano, M.; Siracusa, V.; Munari, A.; Dalla Rosa, M. Biodegradable Long Chain Aliphatic Polyesters Containing Ether-Linkages: Synthesis, Solid-State, and Barrier Properties. Ind. Eng. Chem. Res. 2014, 53, 10965-10973. [CrossRef]

43. Guidotti, G.; Soccio, M.; Siracusa, V.; Gazzano, M.; Salatelli, E.; Munari, A.; Lotti, N. Novel Random PBS-Based Copolymers Containing Aliphatic Side Chains for Sustainable Flexible Food Packaging. Polymers 2017, 9, 724. [CrossRef]

44. Robertson, G.L. Optical, Mechanical and Barrier Properties of Thermoplastics Polymers. In Food Packaging-Principles and Practice, 3rd ed.; CRC Press: Boca Raton, FL, USA, 2013; pp. 91-130.

45. Mensitieri, G.; Di Maio, E.; Buonocore, G.G.; Nedi, I.; Oliviero, M.; Sansone, L.; Iannace, S. Processing and shelf life issues of selected food packaging materials and structures from renewable resources. Trends Food Sci. Technol. 2011, 22, 72-80. [CrossRef] 